\title{
Homogenization of an elastic double-porosity medium with imperfect interface via the periodic unfolding method
}

Patrizia Donato ${ }^{1}$ and lulian Ţenţea ${ }^{2^{*}}$

\section{"Correspondence:}

iuliantentea@yahoo.com

${ }^{2}$ Simion Stoilow Institute of

Mathematics of the Romanian

Academy, 21 Calea Grivitei Street,

Bucharest, 010702, Romania

Full list of author information is

available at the end of the article

\begin{abstract}
We study an $\varepsilon$-periodic model of a medium with double porosity which consists of two components, one of them being connected. We assume that the elasticity of the medium in the inclusion is of order $\varepsilon^{2}$ and also, on the interface between the two components, we consider a jump of the displacement vector condition, proportional to the stress tensor which is continuous. The aim of the paper is to prove the convergence of the homogenization process using the periodic unfolding method.
\end{abstract}

Keywords: homogenization; elasticity; double porosity; interface jumps

\section{Introduction}

This paper deals with the homogenization of a double porosity model in elasticity describing a medium occupying an open set $\Omega$ in $\mathbb{R}^{N}$ which consists of two components, one of them being connected and the second one disconnected.

More exactly, we suppose that $\Omega$ is the union of two open subsets $\Omega_{1}^{\varepsilon}$ and $\Omega_{2}^{\varepsilon}$ and their common boundary $\Gamma^{\varepsilon}$, and we consider the problem

$$
\left\{\begin{array}{l}
-\frac{\partial \sigma_{i j}^{\alpha \varepsilon}}{\partial x_{j}}=g_{i} \quad \text { in } \Omega_{\alpha}^{\varepsilon}, \alpha \in\{1,2\}, \\
\sigma_{i j}^{1 \varepsilon} n_{j}=\sigma_{i j}^{2 \varepsilon} n_{j} \text { on } \Gamma_{\varepsilon}, \\
\sigma_{i j}^{1 \varepsilon} n_{j}=\varepsilon h_{\varepsilon}\left(u_{i}^{2 \varepsilon}-u_{i}^{1 \varepsilon}\right) \text { on } \Gamma_{\varepsilon}, \\
u^{1 \varepsilon}=0 \text { on } \partial \Omega
\end{array}\right.
$$

where $n$ is the outward unit normal to $\Omega_{1}^{\varepsilon}$. The set $\Omega_{2}^{\varepsilon}$ is a disconnected union of $\varepsilon$-periodic open sets. We suppose that the elasticity tensor, which defines the strains $\sigma$, is of order 1 in $\Omega_{1}^{\varepsilon}$ and of order $\varepsilon^{2}$ in the inclusions $\Omega_{2}^{\varepsilon}$.

The jump conditions on the surface model in fact a layer of a soft material that surrounds the particles; the layer is here modeled as a surface, thus not only the tangential, but also the normal component of the displacement can have a jump.

Using the periodic unfolding method, we prove some convergence results and describe the homogenized problems.

More precisely, first, in Theorem 11 we describe the homogenized problem in the variables $x$ and $y$, as is usually done. Then, in Theorem 12 we identify the homogenized prob-

\section{然 Springer}

๑2013 Donato and Țenţea; licensee Springer. This is an Open Access article distributed under the terms of the Creative Commons Attribution License (http://creativecommons.org/licenses/by/2.0), which permits unrestricted use, distribution, and reproduction in any medium, provided the original work is properly cited. 
lem in $\Omega$, and we show that

$$
\begin{aligned}
& \tilde{u}^{1 \varepsilon} \rightarrow\left|Y_{1}\right| \cdot u^{1} \quad \text { weakly in } L^{2}(\Omega)^{N}, \\
& \tilde{u}^{2 \varepsilon} \rightarrow\left|Y_{2}\right| \cdot u^{1}+g_{l} \cdot q^{l} \quad \text { weakly in } L^{2}(\Omega)^{N},
\end{aligned}
$$

where, for $\alpha \in\{1,2\}, \tilde{u}^{\alpha \varepsilon}$ is the extension by zero of $u^{\alpha \varepsilon}$ to the whole $\Omega$ and $u^{1}$ is the unique solution of the problem

$$
\left\{\begin{array}{l}
-\frac{\partial}{\partial x_{j}}\left(a_{i j k h}^{*} \frac{\partial u_{k}^{1}}{\partial x_{h}}\right)=g_{i} \text { in } \Omega, \\
u=0 \quad \text { on } \partial \Omega .
\end{array}\right.
$$

The homogenized tensor $A^{*}$ is the same as that obtained for the usual problem stated in the perforated domain $\Omega_{1}^{\varepsilon}$ with a homogeneous Neumann boundary condition.

The presence of the tensor of order $\varepsilon^{2}$ in the second component does not contribute to the homogenized tensor $A^{*}$. However, it gives rise to the additional term $g_{l} \cdot q^{l}$ in the limit of $\tilde{u}^{2 \varepsilon}$, where $q_{i}^{l}=\int_{Y_{2}} w_{2 i}^{l} \mathrm{~d} y$ for $i, l=1, \ldots, N$ and $w_{2}^{l} \in H^{1}\left(Y_{2}\right)^{N}$ is the unique solution of the cell problem

$$
\left\{\begin{array}{l}
-\frac{\partial}{\partial y_{j}}\left(a_{i j k h} \frac{\partial w_{2 k}^{l}}{\partial y_{h}}\right)=\delta_{i l} \quad \text { in } Y_{2}, \\
a_{i j k h} \frac{\partial w_{2 k}^{l}}{\partial y_{h}} n_{j}=h w_{2 i}^{l} \quad \text { on } \Gamma .
\end{array}\right.
$$

The periodic unfolding method was introduced by Cioranescu, Damlamian and Griso in [1] (see [2] for a general presentation) and later it was extended to periodically perforated domains by Cioranescu, Damlamian, Donato, Griso and Zaki in [3] and [4]. In [5] Donato et al. use the periodic unfolding method for a two-component domain similar to the one considered in this paper. Hence, they introduce two unfolding operators. The first one is denoted by $\mathcal{T}_{1}^{\varepsilon}$ and deals with functions defined on $\Omega_{1}^{\varepsilon}$ which is the same operator introduced in [1]. The second one concerns the functions defined on $\Omega_{2}^{\varepsilon}$ and is denoted by $\mathcal{T}_{2}^{\varepsilon}$. Similar properties for $\mathcal{T}_{2}^{\varepsilon}$ and also the relations between the two operators and, in particular, the properties of their traces on the common boundary are investigated.

Here, we adapt the ideas therein to our problem and, because the elasticity tensor in the inclusion is of order $\varepsilon^{2}$, we define a suitable functional space endowed with an adequate norm (see Remark 2). Also, when we apply the unfolding method, we need to construct proper test functions, which is a key step in obtaining the homogenized problem.

The pioneering paper for the heat diffusion in a two-component domain with similar jump conditions on the interface is due to Auriault and Ene [6] (see also [7], where the results were proved using asymptotic expansions). Later, in [8], Ene and Poliševski gave a rigorous proof of the convergence for one of the cases studied in [6] using the two-scale convergence method introduced in [9] and developed in [10]. Several cases of the same problem were treated by Monsurrò in [11] and Donato and Monsurrò in [12]. Successively, Donato et al. in [5] treated the problem by the periodic unfolding method. In [13] Poliševski added $\varepsilon^{2}$ to the diffusion matrix in the inclusions.

In the present paper, we extend the results of [13] to the case of the linearized elasticity. The main difficulty when applying the periodic unfolding method consists in finding suitable test functions adapted to our elasticity tensor and to the interface term appearing 
in the variational formulation. Let us mention that a similar model is treated via asymptotic expansions by Smyshlyaev in [14], where, in the inclusions, the tensor is of the form $\varepsilon^{2} A(x / \varepsilon)+B(x / \varepsilon)$ with $B$ being only non-negative. Other related elasticity problems were studied by Damlamian, Cioranescu and Orlik in [15] and [16].

For classical results about the homogenization of the linearized elasticity system in composites media, see, for instance, [17-20] and references therein. For the case of periodically perforated domains, we refer to Léné [21] (see also [22]).

\section{The domain}

Let $\Omega$ be an open bounded subset of $\mathbb{R}^{N}(N \geq 2)$ with a Lipschitz continuous boundary $\partial \Omega$ and $Y=(0,1)^{N}$ the unit cube in $\mathbb{R}^{N}$. We suppose that $Y_{2}$ is a subset of $Y$ such that $\bar{Y}_{2} \subset Y$ and its boundary $\Gamma$ is also Lipschitz continuous. Moreover, we define $Y_{1}=Y \backslash \bar{Y}_{2}$. One can see that repeating $Y$ by periodicity, the union of all $\bar{Y}_{1}$ is a connected domain in $\mathbb{R}^{N}$ which will be denoted by $\mathbb{R}_{1}^{N}$. Furthermore, $\mathbb{R}_{2}^{N}=\mathbb{R}^{N} \backslash \mathbb{R}_{1}^{N}$.

In the following, the parameter $\varepsilon \in(0,1)$ takes its values in a sequence of real numbers which, in the homogenizing process, will tend to zero. For each $k \in \mathbb{Z}^{N}$, we define $Y^{k}=$ $k+Y$ and $Y_{\alpha}^{k}=k+Y_{\alpha}$, where $\alpha \in\{1,2\}$. We also define, for each $\varepsilon$,

$$
\mathbb{Z}_{\varepsilon}=\left\{k \in \mathbb{Z}^{N}: \varepsilon \bar{Y}_{2}^{k} \subset \Omega\right\},
$$

and we set (see Figure 1)

$$
\Omega_{2}^{\varepsilon}=\bigcup_{k \in \mathbb{Z}_{\varepsilon}}\left(\varepsilon Y_{2}^{k}\right) \quad \text { and } \quad \Omega_{1}^{\varepsilon}=\Omega \backslash \bar{\Omega}_{2}^{\varepsilon}
$$

The boundary of $\Omega_{2}^{\varepsilon}$ will be denoted by $\Gamma_{\varepsilon}$ and $n$ will be the normal on $\Gamma_{\varepsilon}$ exterior to $\Omega_{1}^{\varepsilon}$.

We introduce now, for each $\varepsilon$, the jump factor $h_{\varepsilon}(x)=h(x / \varepsilon)$ and the fourth-order elasticity tensor $A^{\varepsilon}$ defined by

$$
A^{\varepsilon}(x)= \begin{cases}A(x / \varepsilon) & \text { in } Y_{1}, \\ \varepsilon^{2} A(x / \varepsilon) & \text { in } Y_{2},\end{cases}
$$

where $h \in L^{\infty}(\Gamma)$ and the components $a_{i j k h} \in L^{\infty}(Y)$ are smooth, real, $Y$-periodic functions, with the property that there exists $\lambda>0$ such that

$$
h(y) \geq \lambda \quad \text { and } \quad a_{i j k h}(y) \xi_{i j} \xi_{k h} \geq \lambda \xi_{i j} \xi_{i j}
$$

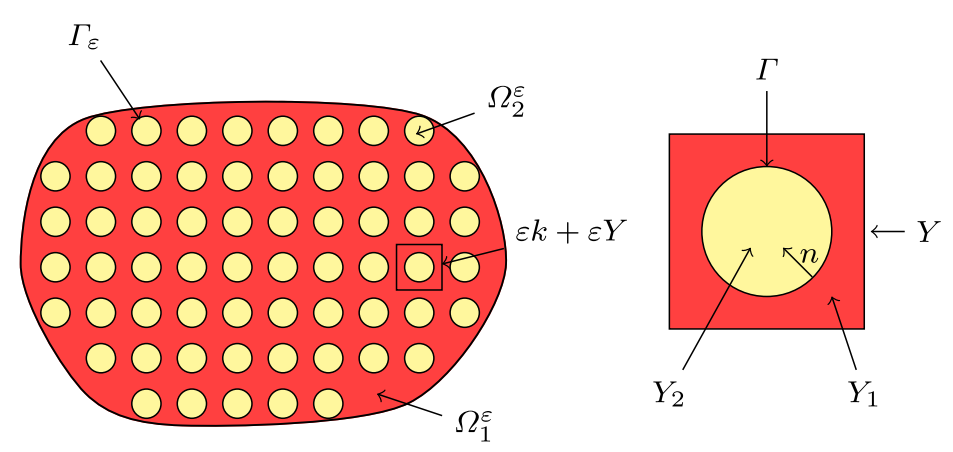

Figure 1 The domain $\Omega=\bar{\Omega}_{1}^{\varepsilon} \cup \Omega_{2}^{\varepsilon}$ and the period cell $Y=(0,1)^{N}$. 
for any $y \in Y$ and any symmetric tensor $\xi_{i j}$. We also assume the symmetry condition for the elasticity tensor, namely

$$
a_{i j k h}=a_{j i k h}=a_{i j h k}=a_{k h i j}
$$

Finally, for $\alpha \in\{1,2\}$ and a function $u^{\alpha \varepsilon}$ defined on $\Omega_{\alpha}^{\varepsilon}$, we denote the stress tensors by $\sigma_{i j}^{\alpha \varepsilon}=a_{i j k h}^{\varepsilon} e_{k h}\left(u^{\alpha \varepsilon}\right)$, where $e_{k h}\left(u^{\alpha \varepsilon}\right)$ represent the components of the deformation tensor defined for any function $v$ by

$$
e_{k h}(v)=\frac{1}{2}\left(\frac{\partial v_{k}}{\partial x_{h}}+\frac{\partial v_{h}}{\partial x_{k}}\right) \text {. }
$$

One can see that due to the symmetry of the deformation tensor, we have $\sigma_{i j}^{\alpha \varepsilon}=a_{i j k h}^{\varepsilon} \frac{\partial u_{k}^{\alpha \varepsilon}}{\partial x_{h}}$.

For a general exposition of the elasticity theory, we refer, for instance, to Ciarlet [23], Duvaut and Lions [24] (see also [25]).

\section{The problem}

Our goal is to describe the asymptotic behavior, as $\varepsilon \rightarrow 0$, of the problem

$$
-\frac{\partial \sigma_{i j}^{\alpha \varepsilon}}{\partial x_{j}}=g_{i} \quad \text { in } \Omega_{\alpha}^{\varepsilon}, \alpha \in\{1,2\},
$$

where $g_{i}$ are the components of a vector field $g \in L^{2}(\Omega)^{N}$ which represent the given body forces together with the boundary conditions

$$
\begin{aligned}
& \sigma_{i j}^{1 \varepsilon} n_{j}=\sigma_{i j}^{2 \varepsilon} n_{j} \quad \text { on } \Gamma_{\varepsilon}, \\
& \sigma_{i j}^{1 \varepsilon} n_{j}=\varepsilon h_{\varepsilon}\left(u_{i}^{2 \varepsilon}-u_{i}^{1 \varepsilon}\right) \text { on } \Gamma_{\varepsilon}, \\
& u^{1 \varepsilon}=0 \quad \text { on } \partial \Omega .
\end{aligned}
$$

Remark 1 Everywhere in this paper we use the Einstein summation convention, except for the cases where we mention that some product $u_{i} v_{i}$ does not follow this convention. Also, if there is no mention of the contrary, the writing $\left\|v_{i}\right\|^{2}$ will follow the same convention since $\left\|v_{i}\right\|^{2}=\left\|v_{i}\right\| \times\left\|v_{i}\right\|$.

We introduce the space $V_{\varepsilon}=\left\{v \in H^{1}\left(\Omega_{1}^{\varepsilon}\right), v=0\right.$ on $\left.\partial \Omega\right\}$ equipped with the $L^{2}$-norm of the gradient and the Hilbert space

$$
W^{\varepsilon}=V_{\varepsilon}^{N} \times H^{1}\left(\Omega_{2}^{\varepsilon}\right)^{N}
$$

endowed with the scalar product

$$
(u, v)_{W^{\varepsilon}}=\int_{\Omega_{1}^{\varepsilon}} \nabla u_{i}^{1} \nabla v_{i}^{1} \mathrm{~d} x+\varepsilon^{2} \int_{\Omega_{2}^{\varepsilon}} \nabla u_{i}^{2} \nabla v_{i}^{2} \mathrm{~d} x+\varepsilon \int_{\Gamma_{\varepsilon}}\left(u_{i}^{2}-u_{i}^{1}\right)\left(v_{i}^{2}-v_{i}^{1}\right) \mathrm{d} \sigma_{x},
$$

where the elements of any $u \in W^{\varepsilon}$ are denoted by $u=\left(u^{1}, u^{2}\right)$. 
The norm generated by the scalar product (3.6) is given by

$$
\|v\|_{W^{\varepsilon}}^{2}=\left\|\nabla v_{i}^{1}\right\|_{L^{2}\left(\Omega_{2}^{\varepsilon}\right)}^{2}+\varepsilon^{2}\left\|\nabla v_{i}^{2}\right\|_{L^{2}\left(\Omega_{2}^{\varepsilon}\right)}^{2}+\varepsilon\left\|v_{i}^{2}-v_{i}^{1}\right\|_{L^{2}\left(\Gamma_{\varepsilon}\right)}^{2} .
$$

Remark 2 Due to the fact that the elasticity tensor is of order $\varepsilon^{2}$ in the inclusion $\Omega_{2}^{\varepsilon}$, the functional space $W^{\varepsilon}$ and the norm defined by (3.7) are different from those used in [5]. More precisely, in [5] the space used was $H_{\gamma}^{\varepsilon}=V_{\varepsilon} \times H^{1}\left(\Omega_{2}^{\varepsilon}\right)$ and it was equipped with the norm

$$
\|v\|_{H_{\gamma}^{\varepsilon}}^{2}=\left\|\nabla v^{1}\right\|_{L^{2}\left(\Omega_{2}^{\varepsilon}\right)}^{2}+\left\|\nabla v^{2}\right\|_{L^{2}\left(\Omega_{2}^{\varepsilon}\right)}^{2}+\varepsilon^{\gamma}\left\|v^{2}-v^{1}\right\|_{L^{2}\left(\Gamma_{\varepsilon}\right)}^{2},
$$

where $\gamma$ was the order of $\varepsilon$ in the boundary condition (3.3), the case studied in the present paper being $\gamma=1$.

For any $u, v \in W^{\varepsilon}$, we also use the notation

$$
a(u, v)=\sum_{\alpha=1,2} \int_{\Omega_{\alpha}^{\varepsilon}} a_{i j k h}^{\varepsilon} \frac{\partial u_{k}^{\alpha}}{\partial x_{h}} \frac{\partial v_{i}^{\alpha}}{\partial x_{j}} \mathrm{~d} x+\varepsilon \int_{\Gamma_{\varepsilon}} h_{\varepsilon}\left(u_{i}^{2}-u_{i}^{1}\right)\left(v_{i}^{2}-v_{i}^{1}\right) \mathrm{d} \sigma_{x},
$$

and we introduce the following variational formulation of problem (3.1):

Find $u^{\varepsilon} \in W^{\varepsilon}$ such that

$$
a\left(u_{\varepsilon}, v\right)=\int_{\Omega_{1}^{\varepsilon}} g_{i} v_{i}^{1} \mathrm{~d} x+\int_{\Omega_{2}^{\varepsilon}} g_{i} v_{i}^{2} \mathrm{~d} x, \quad \forall v \in W^{\varepsilon}
$$

Theorem 3 For any $\varepsilon \in(0,1)$, problem (3.10) has a unique solution $u^{\varepsilon} \in W^{\varepsilon}$. Moreover, there exists a constant $C>0$ independent of $\varepsilon$ such that, for $\alpha \in\{1,2\}$ and each $i=1, \ldots, N$, we have

$$
\begin{aligned}
& \left\|u_{i}^{\varepsilon \alpha}\right\|_{L^{2}\left(\Omega_{\alpha}^{\varepsilon}\right)} \leq C, \quad\left\|\nabla u_{i}^{1 \varepsilon}\right\|_{L^{2}\left(\Omega_{1}^{\varepsilon}\right)} \leq C \quad \text { and } \quad \varepsilon\left\|\nabla u_{i}^{2 \varepsilon}\right\|_{L^{2}\left(\Omega_{2}^{\varepsilon}\right)} \leq C, \\
& \left\|u_{i}^{2 \varepsilon}-u_{i}^{1 \varepsilon}\right\|_{L^{2}\left(\Gamma_{\varepsilon}\right)} \leq C \varepsilon^{-1 / 2}
\end{aligned}
$$

Proof The result is proved by applying the Lax-Milgram theorem. The coerciveness of the form $a(\cdot, \cdot)$ can be easily shown using definition (3.9) and the properties (2.4) which give

$$
\begin{aligned}
a(v, v) & \geq \lambda\left(\int_{\Omega_{1}^{\varepsilon}} \nabla v_{i}^{1} \nabla v_{i}^{1} \mathrm{~d} x+\varepsilon^{2} \int_{\Omega_{2}^{\varepsilon}} \nabla v_{i}^{2} \nabla v_{i}^{2} \mathrm{~d} x+\varepsilon \int_{\Gamma_{\varepsilon}}\left(v_{i}^{2}-v_{i}^{1}\right)^{2} \mathrm{~d} \sigma_{x}\right) \\
& =\lambda\|v\|_{W^{\varepsilon}}^{2} .
\end{aligned}
$$

Let us prove now the continuity of the right-hand side of (3.10). Applying the CauchySchwarz inequality, we obtain

$$
\begin{aligned}
\int_{\Omega_{1}^{\varepsilon}} g_{i} v_{i}^{1} \mathrm{~d} x+\int_{\Omega_{2}^{\varepsilon}} g_{i} v_{i}^{2} \mathrm{~d} x & \leq\left\|g_{i}\right\|_{L^{2}\left(\Omega_{1}^{\varepsilon}\right)}\left\|v_{i}^{1}\right\|_{L^{2}\left(\Omega_{1}^{\varepsilon}\right)}+\left\|g_{i}\right\|_{L^{2}\left(\Omega_{2}^{\varepsilon}\right)}\left\|v_{i}^{2}\right\|_{L^{2}\left(\Omega_{2}^{\varepsilon}\right)} \\
& \leq C\left(\left\|v_{i}^{1}\right\|_{L^{2}\left(\Omega_{1}^{\varepsilon}\right)}+\left\|v_{i}^{2}\right\|_{L^{2}\left(\Omega_{2}^{\varepsilon}\right)}\right) .
\end{aligned}
$$


It remains to show that $\left\|v_{i}^{1}\right\|_{L^{2}\left(\Omega_{1}^{\varepsilon}\right)}+\left\|v_{i}^{2}\right\|_{L^{2}\left(\Omega_{2}^{\varepsilon}\right)} \leq C\|v\|_{W^{\varepsilon}}$. It is known (see $\left.[8,11]\right)$ that there exists some constant $C>0$ independent of $\varepsilon$ such that for each $i=1, \ldots, N$,

$$
\begin{aligned}
& \left\|v_{i}^{2}\right\|_{L^{2}\left(\Omega_{2}^{\varepsilon}\right)} \leq C\left(\varepsilon\left\|\nabla v_{i}^{2}\right\|_{L^{2}\left(\Omega_{2}^{\varepsilon}\right)}+\varepsilon^{1 / 2}\left\|v_{i}^{2}\right\|_{L^{2}\left(\Gamma_{\varepsilon}\right)}\right), \\
& \varepsilon^{1 / 2}\left\|v_{i}^{1}\right\|_{L^{2}\left(\Gamma_{\varepsilon}\right)} \leq C\left(\varepsilon\left\|\nabla v_{i}^{1}\right\|_{L^{2}\left(\Omega_{1}^{\varepsilon}\right)}+\left\|v_{i}^{1}\right\|_{L^{2}\left(\Omega_{1}^{\varepsilon}\right)}\right), \\
& \left\|v_{i}^{1}\right\|_{L^{2}\left(\Omega_{1}^{\varepsilon}\right)} \leq C\left\|\nabla v_{i}^{1}\right\|_{L^{2}\left(\Omega_{1}^{\varepsilon}\right)} .
\end{aligned}
$$

From (3.13) we have

$$
\left\|v_{i}^{2}\right\|_{L^{2}\left(\Omega_{2}^{\varepsilon}\right)} \leq C\left(\varepsilon\left\|\nabla v_{i}^{2}\right\|_{L^{2}\left(\Omega_{2}^{\varepsilon}\right)}+\varepsilon^{1 / 2}\left\|v_{i}^{2}-v_{i}^{1}\right\|_{L^{2}\left(\Gamma_{\varepsilon}\right)}+\varepsilon^{1 / 2}\left\|v_{i}^{1}\right\|_{L^{2}\left(\Gamma_{\varepsilon}\right)}\right)
$$

Using now (3.14) and (3.15), we obtain

$$
\left\|v_{i}^{1}\right\|_{L^{2}\left(\Omega_{1}^{\varepsilon}\right)}+\left\|v_{i}^{2}\right\|_{L^{2}\left(\Omega_{2}^{\varepsilon}\right)} \leq C\|v\|_{W^{\varepsilon}}
$$

In order to give now the a priori estimates for the solution of (3.10), we choose $v=u^{\varepsilon}$ in (3.10). Using the coerciveness of $a(\cdot, \cdot)$, the Cauchy-Schwarz inequality and (3.17), we find $C>0$ independent of $\varepsilon$ such that

$$
\begin{aligned}
\lambda\left\|u^{\varepsilon}\right\|_{W^{\varepsilon}}^{2} & \leq a\left(u^{\varepsilon}, u^{\varepsilon}\right)=\int_{\Omega_{1}^{\varepsilon}} g_{i} v_{i}^{1} \mathrm{~d} x+\int_{\Omega_{2}^{\varepsilon}} g_{i} v_{i}^{2} \mathrm{~d} x \\
& \leq\left\|g_{i}\right\|_{L^{2}(\Omega)}\left(\left\|v_{i}^{1}\right\|_{L^{2}\left(\Omega_{1}^{\varepsilon}\right)}+\left\|v_{i}^{2}\right\|_{L^{2}\left(\Omega_{2}^{\varepsilon}\right)}\right) \leq C\left\|u^{\varepsilon}\right\|_{W^{\varepsilon}} .
\end{aligned}
$$

Therefore

$$
\left\{u^{\varepsilon}\right\}_{\varepsilon} \text { is bounded in } W^{\varepsilon} \text {. }
$$

Moreover, the definition of the norm in $W^{\varepsilon}$ implies that for $\alpha \in\{1,2\}$, estimates (3.11) and (3.12) hold.

\section{Periodic unfolding operators in two-component domain}

In this section we present the definitions of the unfolding operators for a two-component domain introduced by Donato et al. in [5] and their main properties. We will prove only the results that bring some other properties than those already proved in the paper quoted above. The important characteristic of these operators is that they map functions defined on the oscillating domains $\Omega_{1}^{\varepsilon}$ and $\Omega_{2}^{\varepsilon}$ into functions defined on the fixed domains $\Omega \times Y_{1}$ and $\Omega \times Y_{2}$ respectively.

In the following, for $x \in \mathbb{R}^{N}$, we denote by $[x]_{Y}$ its integer part $k \in \mathbb{Z}^{N}$ such that $x-[x]_{Y} \in$ $Y$ and set

$$
\{x\}_{Y}=x-[x]_{Y} \text { for a.e. } x \in \mathbb{R}^{N} .
$$

Then, for almost every $x \in \mathbb{R}^{N}$,

$$
x=\varepsilon\left(\left[\frac{x}{\varepsilon}\right]_{Y}+\left\{\frac{x}{\varepsilon}\right\}_{Y}\right) .
$$




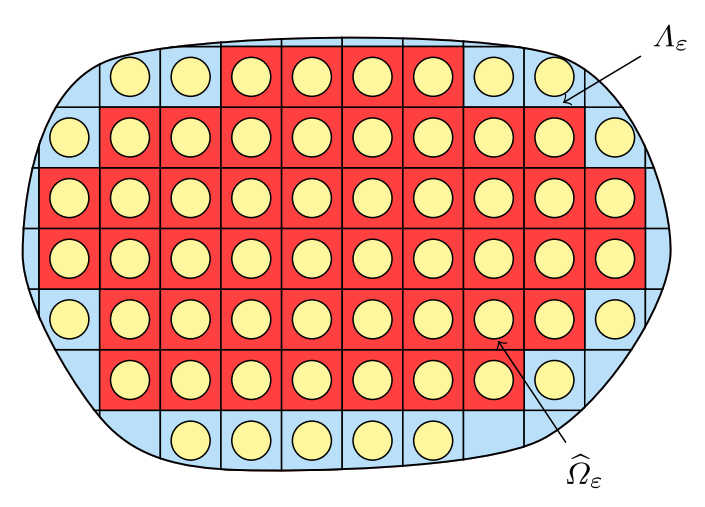

Figure 2 The sets $\widehat{\Omega}_{\varepsilon}$ and $\Lambda_{\varepsilon}$.

We introduce now the sets (see Figure 2)

$$
\begin{aligned}
& \widehat{\mathbb{Z}}_{\varepsilon}=\left\{k \in \mathbb{Z}^{N}: \varepsilon Y^{k} \subset \Omega\right\}, \quad \widehat{\Omega}_{\varepsilon}=\operatorname{int} \bigcup_{k \in \widehat{\mathbb{Z}}_{\varepsilon}}\left(\varepsilon \bar{Y}^{k}\right), \quad \Lambda_{\varepsilon}=\Omega \backslash \widehat{\Omega}_{\varepsilon}, \\
& \widehat{\Omega}_{\alpha}^{\varepsilon}=\bigcup_{k \in \widehat{\mathbb{Z}}_{\varepsilon}}\left(\varepsilon Y_{\alpha}^{k}\right), \quad \Lambda_{\alpha}^{\varepsilon}=\Omega_{\alpha}^{\varepsilon} \backslash \widehat{\Omega}_{\alpha}^{\varepsilon}, \quad \widehat{\Gamma}_{\varepsilon}=\partial \widehat{\Omega}_{2}^{\varepsilon} .
\end{aligned}
$$

For a given set $D \subset \mathbb{R}^{N}$ and $v \in L^{1}(D)$, we also denote $\langle v\rangle_{D}=\frac{1}{|D|} \int_{D} v(y) \mathrm{d} y$. If there is no place for confusion, we will simply use $\langle v\rangle$ instead of $\langle v\rangle_{Y}$. If $v$ is a function defined on $\Omega_{\alpha}^{\varepsilon}$, $\alpha \in\{1,2\}$, then $\tilde{v}$ will denote the extension by zero to the whole $\Omega$. Furthermore, $\chi_{\alpha}$ will be the characteristic function of $\Omega_{\alpha}$ for each $\alpha \in\{1,2\}$, and we introduce the spaces

$$
\begin{aligned}
& H_{\text {per }}^{1}\left(Y_{\alpha}\right)=\left\{v \in H_{\text {loc }}^{1}\left(\mathbb{R}_{\alpha}^{N}\right): v \text { is } Y \text {-periodic }\right\}, \\
& \tilde{H}_{\text {per }}^{1}\left(Y_{\alpha}\right)=\left\{v \in H_{\text {per }}^{1}\left(Y_{\alpha}\right):\langle v\rangle_{Y}=0\right\} .
\end{aligned}
$$

Definition 4 For any Lebesgue measurable function $\varphi$ on $\Omega_{\alpha}^{\varepsilon}, \alpha \in\{1,2\}$, we define the periodic unfolding operator by the formula

$$
\mathcal{T}_{\alpha}^{\varepsilon}(\varphi)(x, y)= \begin{cases}\varphi\left(\varepsilon\left[\frac{x}{\varepsilon}\right]_{Y}+\varepsilon y\right) & \text { for a.e. }(x, y) \in \widehat{\Omega}_{\varepsilon} \times Y_{\alpha} \\ 0 & \text { for a.e. }(x, y) \in \Lambda_{\varepsilon} \times Y_{\alpha}\end{cases}
$$

Remark 5 If $\varphi$ is a function defined in $\Omega$, then, for the sake of simplicity, we write $\mathcal{T}_{\alpha}^{\varepsilon}(\varphi)$ instead of $\mathcal{T}_{\alpha}^{\varepsilon}\left(\left.\varphi\right|_{\Omega_{\alpha}^{\varepsilon}}\right)$.

Proposition 6 For $p \in[1, \infty)$ and $\alpha \in\{1,2\}$, the operators $\mathcal{T}_{\alpha}^{\varepsilon}$ are continuous from $L^{p}\left(\Omega_{\alpha}^{\varepsilon}\right)$ to $L^{p}\left(\Omega \times Y_{\alpha}\right)$. Moreover,

(i) if $\varphi$ and $\psi$ are two Lebesgue measurable functions on $\Omega_{\alpha}^{\varepsilon}$, one has $\mathcal{T}_{\alpha}^{\varepsilon}(\varphi \psi)=\mathcal{T}_{\alpha}^{\varepsilon}(\varphi) \cdot \mathcal{T}_{\alpha}^{\varepsilon}(\psi)$,

(ii) for every $\varphi \in L^{1}\left(\Omega_{\alpha}^{\varepsilon}\right)$,

$$
\int_{\Omega \times Y_{\alpha}} \mathcal{T}_{\alpha}^{\varepsilon}(\varphi)(x, y) \mathrm{d} x \mathrm{~d} y=\int_{\widehat{\Omega}_{\alpha}^{\varepsilon}} \varphi(x) \mathrm{d} x=\int_{\Omega_{\alpha}^{\varepsilon}} \varphi(x) \mathrm{d} x-\int_{\Lambda_{\varepsilon}} \varphi(x) \mathrm{d} x,
$$


(iii) $\left\|\mathcal{T}_{\alpha}^{\varepsilon}(\varphi)\right\|_{L^{p}\left(\Omega \times Y_{\alpha}\right)} \leq\|\varphi\|_{L^{p}\left(\Omega_{\alpha}^{\varepsilon}\right)}$ for every $\varphi \in L^{p}\left(\Omega_{\alpha}^{\varepsilon}\right)$,

(iv) $\mathcal{T}_{\alpha}^{\varepsilon}(\varphi) \longrightarrow \varphi$ strongly in $L^{p}\left(\Omega \times Y_{\alpha}\right)$ for $\varphi \in L^{p}(\Omega)$,

(v) if $\left\{\varphi_{\varepsilon}\right\}_{\varepsilon} \subset L^{p}(\Omega)$ is a sequence such that $\varphi_{\varepsilon} \longrightarrow \varphi$ strongly in $L^{p}(\Omega)$, then $\mathcal{T}_{\alpha}^{\varepsilon}\left(\varphi_{\varepsilon}\right) \longrightarrow \varphi$ strongly in $L^{p}\left(\Omega \times Y_{\alpha}\right)$,

(vi) if $\varphi \in L^{p}\left(Y_{\alpha}\right)$ is $Y$-periodic and $\varphi_{\varepsilon}(x)=\varphi(x / \varepsilon)$, then $\mathcal{T}_{\alpha}^{\varepsilon}\left(\varphi_{\varepsilon}\right) \longrightarrow \varphi$ strongly in $L^{p}\left(\Omega \times Y_{\alpha}\right)$,

(vii) if $\varphi_{\varepsilon} \in L^{p}\left(\Omega_{\alpha}^{\varepsilon}\right)$ satisfies $\left\|\varphi_{\varepsilon}\right\|_{L^{p}\left(\Omega_{\alpha}^{\varepsilon}\right)} \leq C$ and $\mathcal{T}_{\alpha}^{\varepsilon}\left(\varphi_{\varepsilon}\right) \rightarrow \hat{\varphi}$ weakly in $L^{p}\left(\Omega \times Y_{\alpha}\right)$, then $\tilde{\varphi}_{\varepsilon} \rightarrow\left|Y_{\alpha}\right|\langle\hat{\varphi}\rangle_{\alpha}$ weakly in $L^{p}(\Omega)$,

(viii) if $\varphi \in W^{1, p}\left(\Omega_{\alpha}^{\varepsilon}\right)$, then $\nabla_{y}\left(\mathcal{T}_{\alpha}^{\varepsilon}(\varphi)\right)=\varepsilon \mathcal{T}_{\alpha}^{\varepsilon}(\nabla \varphi)$ and $\mathcal{T}_{\alpha}^{\varepsilon}(\varphi)$ belongs to $L^{2}\left(\Omega ; W^{1, p}\left(Y_{\alpha}\right)\right)$.

Lemma 7 If $u^{\varepsilon}=\left(u^{1 \varepsilon}, u^{2 \varepsilon}\right)$ is a sequence in $W^{\varepsilon}$, then, for each $i=1, \ldots, N$,

$$
\frac{1}{\varepsilon} \int_{\Omega \times \Gamma}\left|\mathcal{T}_{2}^{\varepsilon}\left(u_{i}^{2 \varepsilon}\right)-\mathcal{T}_{1}^{\varepsilon}\left(u_{i}^{1 \varepsilon}\right)\right|^{2} \mathrm{~d} x \mathrm{~d} \sigma_{y} \leq \int_{\Gamma_{\varepsilon}}\left|u_{i}^{2 \varepsilon}-u_{i}^{1 \varepsilon}\right|^{2} \mathrm{~d} \sigma_{x} .
$$

Remark 8 From (3.11), (3.12) and Proposition 6(iii), it is easy to see that for each $i=$ $1, \ldots, N$

$$
\begin{aligned}
& \left\|\mathcal{T}_{1}^{\varepsilon}\left(\nabla u_{i}^{1 \varepsilon}\right)\right\|_{L^{2}\left(\Omega \times Y_{1}\right)} \leq C, \\
& \varepsilon\left\|\mathcal{T}_{2}^{\varepsilon}\left(\nabla u_{i}^{2 \varepsilon}\right)\right\|_{L^{2}\left(\Omega \times Y_{2}\right)} \leq C, \\
& \left\|\mathcal{T}_{2}^{\varepsilon}\left(u_{i}^{2 \varepsilon}\right)-\mathcal{T}_{1}^{\varepsilon}\left(u_{i}^{1 \varepsilon}\right)\right\|_{L^{2}(\Omega \times \Gamma)} \leq C .
\end{aligned}
$$

Lemma 9 If $\varphi \in \mathcal{D}(\Omega)^{N}$ and $u^{\varepsilon}=\left(u^{1 \varepsilon}, u^{2 \varepsilon}\right) \in W^{\varepsilon}$, then, for $\varepsilon$ small enough, for each $i=$ $1, \ldots, N$, we have

$$
\varepsilon \int_{\Gamma_{\varepsilon}} h_{\varepsilon}\left(u_{i}^{2 \varepsilon}-u_{i}^{1 \varepsilon}\right) \varphi_{i} \mathrm{~d} \sigma_{x}=\int_{\Omega \times \Gamma} h(y)\left(\mathcal{T}_{2}^{\varepsilon}\left(u_{i}^{2 \varepsilon}\right)-\mathcal{T}_{1}^{\varepsilon}\left(u_{i}^{1 \varepsilon}\right)\right) \mathcal{T}_{1}^{\varepsilon}\left(\varphi_{i}\right) \mathrm{d} x \mathrm{~d} \sigma_{y} .
$$

Theorem 10 Let $u^{\varepsilon}=\left(u^{1 \varepsilon}, u^{2 \varepsilon}\right)$ be a bounded sequence in $W^{\varepsilon}$. Then there exist a subsequence (still denoted by $\varepsilon$ ), $u^{1} \in H_{0}^{1}(\Omega)^{N}, \hat{u}^{1} \in L^{2}\left(\Omega ; H_{\mathrm{per}}^{1}\left(Y_{1}\right)\right)^{N}$ and $\hat{u}^{2} \in L^{2}\left(\Omega ; H^{1}\left(Y_{1}\right)\right)^{N}$ such that for each $i=1, \ldots, N$,

$$
\begin{aligned}
& \mathcal{T}_{1}^{\varepsilon}\left(u_{i}^{1 \varepsilon}\right) \longrightarrow u_{i}^{1} \quad \text { strongly in } L^{2}\left(\Omega ; H^{1}\left(Y_{1}\right)\right), \\
& \mathcal{T}_{1}^{\varepsilon}\left(\nabla u_{i}^{1 \varepsilon}\right) \rightarrow \nabla u_{i}^{1}+\nabla_{y} \hat{u}_{i}^{1} \quad \text { weakly in } L^{2}\left(\Omega \times Y_{1}\right), \\
& \mathcal{T}_{2}^{\varepsilon}\left(u_{i}^{2 \varepsilon}\right) \rightarrow \hat{u}_{i}^{2} \quad \text { weakly in } L^{2}\left(\Omega ; H^{1}\left(Y_{2}\right)\right), \\
& \varepsilon \mathcal{T}_{2}^{\varepsilon}\left(\nabla u_{i}^{2 \varepsilon}\right) \rightarrow \nabla_{y} \hat{u}_{i}^{2} \quad \text { weakly in } L^{2}\left(\Omega \times Y_{2}\right),
\end{aligned}
$$

where $\left\langle\hat{u}_{i}^{1}\right\rangle_{\Gamma}=0$ for almost every $x \in \Omega$. Furthermore,

$$
Z_{1}^{\varepsilon}=\frac{1}{\varepsilon}\left[\mathcal{T}_{1}^{\varepsilon}\left(u_{i}^{1 \varepsilon}\right)-\left\langle\mathcal{T}_{1}^{\varepsilon}\left(u_{i}^{1 \varepsilon}\right)\right\rangle_{\Gamma}\right] \rightarrow y_{\Gamma} \nabla u_{i}^{1}+\hat{u}_{i}^{1},
$$

weakly in $L^{2}\left(\Omega ; H^{1}\left(Y_{1}\right)\right)$, where $y_{\Gamma}=y-\langle y\rangle_{\Gamma}$.

Proof Convergences (4.3) $)_{1,2}$ and (4.4) have already been proved in [4] and [5] respectively. From (3.17) we get that $\left\|u_{i}^{2 \varepsilon}\right\|_{L^{2}\left(\Omega_{2}^{\varepsilon}\right)} \leq C$ and again Proposition 6(iii) implies that

$$
\left\|\mathcal{T}_{2}^{\varepsilon}\left(u_{i}^{2 \varepsilon}\right)\right\|_{L^{2}\left(\Omega \times Y_{2}\right)} \leq C .
$$


From Proposition 6(viii) and (4.2) 2 ,

$$
\left\|\nabla_{y}\left(\mathcal{T}_{2}^{\varepsilon}\left(u_{i}^{2 \varepsilon}\right)\right)\right\|_{L^{2}\left(\Omega \times Y_{2}\right)}=\left\|\varepsilon \mathcal{T}_{2}^{\varepsilon}\left(\nabla u_{i}^{2 \varepsilon}\right)\right\|_{L^{2}\left(\Omega \times Y_{2}\right)} \leq C .
$$

Hence $\left\{\mathcal{T}_{2}^{\varepsilon}\left(u_{i}^{2 \varepsilon}\right)\right\}$ is bounded in $L^{2}\left(\Omega ; H^{1}\left(Y_{2}\right)\right)$, i.e.,

$$
\left\|\mathcal{T}_{2}^{\varepsilon}\left(u_{i}^{2 \varepsilon}\right)\right\|_{L^{2}\left(\Omega ; H^{1}\left(Y_{2}\right)\right)} \leq C,
$$

which means that there exists $\hat{u}^{2} \in L^{2}\left(\Omega ; H^{1}\left(Y_{2}\right)\right)^{N}$ such that $(4.3)_{3}$ holds. Furthermore, it is easy to check that

$$
\nabla_{y}\left(\mathcal{T}_{2}^{\varepsilon}\left(u_{i}^{2 \varepsilon}\right)\right) \rightarrow \nabla_{y} \hat{u}_{i}^{2} \quad \text { weakly in } L^{2}\left(\Omega ; L^{2}\left(Y_{2}\right)\right),
$$

and the fact that $\nabla_{y}\left(\mathcal{T}_{2}^{\varepsilon}\left(u_{i}^{2 \varepsilon}\right)\right)=\varepsilon \mathcal{T}_{2}^{\varepsilon}\left(\nabla u_{i}^{2 \varepsilon}\right)$ ends the proof of $(4.3)_{4}$.

\section{Homogenization results}

In the following we will use the notation

$$
V=H_{0}^{1}(\Omega)^{N} \times L^{2}\left(\Omega ; H_{\text {per }}^{1}\left(Y_{1}\right)\right)^{N} \times L^{2}\left(\Omega ; H^{1}\left(Y_{2}\right)\right)^{N} .
$$

Theorem 11 If $u^{\varepsilon}=\left(u^{1 \varepsilon}, u^{2 \varepsilon}\right)$ is the solution of problem (3.1), then

$$
\begin{aligned}
& \tilde{u}^{1 \varepsilon} \rightarrow\left|Y_{1}\right| \cdot u^{1} \quad \text { weakly in } L^{2}(\Omega)^{N}, \\
& \tilde{u}^{2 \varepsilon} \rightarrow\left|Y_{2}\right| \cdot\left\langle\left.\hat{u}^{2}\right|_{Y_{2}} \quad \text { weakly in } L^{2}(\Omega)^{N},\right. \\
& \mathcal{T}_{1}^{\varepsilon}\left(u^{1 \varepsilon}\right) \rightarrow u^{1} \quad \text { strongly in } L^{2}\left(\Omega ; H^{1}\left(Y_{1}\right)\right)^{N}, \\
& \mathcal{T}_{2}^{\varepsilon}\left(u^{2 \varepsilon}\right) \rightarrow \hat{u}^{2} \quad \text { weakly in } L^{2}\left(\Omega ; H^{1}\left(Y_{2}\right)\right)^{N}, \\
& \mathcal{T}_{1}^{\varepsilon}\left(e_{k h}\left(u^{1 \varepsilon}\right)\right) \rightarrow e_{k h}\left(u^{1}\right)+e_{k h}^{y}\left(\hat{u}^{1}\right) \quad \text { weakly in } L^{2}\left(\Omega \times Y_{1}\right), \\
& \varepsilon \mathcal{T}_{2}^{\varepsilon}\left(e_{k h}\left(u^{2 \varepsilon}\right)\right) \rightarrow e_{k h}^{y}\left(\hat{u}^{2}\right) \quad \text { weakly in } L^{2}\left(\Omega \times Y_{2}\right),
\end{aligned}
$$

where the triplet $\left(u^{1}, \hat{u}^{1}, \hat{u}^{2}\right) \in V$ with $\left\langle\hat{u}_{i}^{1}\right\rangle_{\Gamma}=0$ for a.e. $x \in \Omega$ is the unique solution of the problem

$$
\begin{aligned}
& \int_{\Omega \times Y_{1}} a_{i j k h}\left(\frac{\partial u_{k}^{1}}{\partial x_{h}}+\frac{\partial \hat{u}_{k}^{1}}{\partial y_{h}}\right)\left(\frac{\partial \varphi_{i}}{\partial x_{j}}+\frac{\partial \Phi_{i}^{1}}{\partial y_{j}}\right) \mathrm{d} x \mathrm{~d} y \\
& \quad+\int_{\Omega \times Y_{2}} a_{i j k h} \frac{\partial \hat{u}_{k}^{2}}{\partial y_{h}} \frac{\partial \Phi_{i}^{2}}{\partial y_{j}} \mathrm{~d} x \mathrm{~d} y+\int_{\Omega \times \Gamma} h\left(\hat{u}_{i}^{2}-u_{i}^{1}\right)\left(\Phi_{i}^{2}-\varphi_{i}\right) \mathrm{d} x \mathrm{~d} \sigma_{y} \\
& =\int_{\Omega \times Y_{1}} g_{i} \varphi_{i} \mathrm{~d} x \mathrm{~d} y+\int_{\Omega \times Y_{2}} g_{i} \Phi_{i}^{2} \mathrm{~d} x \mathrm{~d} y, \quad \forall\left(\varphi, \Phi^{1}, \Phi^{2}\right) \in V .
\end{aligned}
$$

Proof Convergences (5.1) $)_{3-6}$ are a consequence of Theorem 10. From (5.1) $)_{3,4}$ and Proposition 6 (vii), we derive that

$$
\begin{aligned}
& \tilde{u}^{1 \varepsilon} \rightarrow\left|Y_{1}\right| \cdot\left\langle u^{1}\right\rangle_{Y_{1}} \quad \text { weakly in } L^{2}(\Omega)^{N}, \\
& \tilde{u}^{2 \varepsilon} \rightarrow\left|Y_{2}\right| \cdot\left\langle\hat{u}^{2}\right\rangle_{Y_{2}} \quad \text { weakly in } L^{2}(\Omega)^{N} .
\end{aligned}
$$


Since $u^{1}$ is constant with respect to $y$, we deduce that

$$
\tilde{u}^{1 \varepsilon} \rightarrow\left|Y_{1}\right| \cdot u^{1} \quad \text { weakly in } L^{2}(\Omega)^{N} .
$$

To obtain the limit problem, we take as test functions in (3.10)

$$
\begin{aligned}
& v_{i}^{1}(x)=\varphi_{i}(x)+\varepsilon \omega_{i}^{1}(x) \psi_{i}^{1 \varepsilon}(x), \\
& v_{i}^{2}(x)=\omega_{i}^{2}(x) \psi_{i}^{2 \varepsilon}(x),
\end{aligned}
$$

with no summation of repeated index where $\varphi, \omega^{\alpha} \in \mathcal{D}(\Omega)^{N}, \psi^{\alpha} \in H_{\mathrm{per}}^{1}\left(Y_{\alpha}\right)^{N}$ and $\psi^{\alpha \varepsilon}(x)=$ $\psi^{\alpha}(x / \varepsilon)(\alpha \in\{1,2\})$. Everywhere in the sequel, the product $\omega_{i}^{\alpha} \psi_{i}^{\alpha \varepsilon}$ will not follow the Einstein summation convention and will represent the $i$ th component of a vector field.

We obtain

$$
\begin{aligned}
& \int_{\Omega_{1}^{\varepsilon}} a_{i j k h}^{\varepsilon} \frac{\partial u_{k}^{1 \varepsilon}}{\partial x_{h}}\left(\frac{\partial \varphi_{i}}{\partial x_{j}}+\frac{\partial\left(\varepsilon \omega_{i}^{1} \psi_{i}^{1 \varepsilon}\right)}{\partial x_{j}}\right) \mathrm{d} x+\varepsilon \int_{\Omega_{2}^{\varepsilon}} a_{i j k h}^{\varepsilon} \frac{\partial u_{k}^{2 \varepsilon}}{\partial x_{h}} \frac{\partial\left(\varepsilon \omega_{i}^{2} \psi_{i}^{2 \varepsilon}\right)}{\partial x_{j}} \mathrm{~d} x \\
& \quad+\varepsilon \int_{\Gamma_{\varepsilon}} h_{\varepsilon}\left(u_{i}^{2 \varepsilon}-u_{i}^{1 \varepsilon}\right)\left(\omega_{i}^{2} \psi_{i}^{2 \varepsilon}-\varphi_{i}\right) \mathrm{d} \sigma_{x}-\varepsilon^{2} \int_{\Gamma_{\varepsilon}} h_{\varepsilon}\left(u_{i}^{2 \varepsilon}-u_{i}^{1 \varepsilon}\right) \omega_{i}^{1} \psi_{i}^{1 \varepsilon} \mathrm{d} \sigma_{x} \\
& =\int_{\Omega_{1}^{\varepsilon}} g_{i} \varphi_{i} \mathrm{~d} x+\varepsilon \int_{\Omega_{1}^{\varepsilon}} g_{i} \omega_{i}^{1} \psi_{i}^{1 \varepsilon} \mathrm{d} x+\int_{\Omega_{2}^{\varepsilon}} g_{i} \omega_{i}^{2} \psi_{i}^{2 \varepsilon} \mathrm{d} x .
\end{aligned}
$$

Using suggestive notations, this can be written as

$$
I_{1}^{\varepsilon}+I_{2}^{\varepsilon}+I_{3}^{\varepsilon}-I_{4}^{\varepsilon}=I_{5}^{\varepsilon}+I_{6}^{\varepsilon}+I_{7}^{\varepsilon} .
$$

We will now apply the appropriate unfolding operator to each term of (5.6) and pass to the limit as $\varepsilon \rightarrow 0$ in order to obtain the limit problem (5.2).

First, observe that $\varepsilon \omega^{1} \psi^{1 \varepsilon}$ strongly converges to zero in $L^{2}(\Omega)^{N}$. Hence, using Proposition $6(\mathrm{v})$, we obtain

$$
\mathcal{T}_{1}^{\varepsilon}\left(\varepsilon \omega^{1} \psi^{1 \varepsilon}\right) \longrightarrow 0 \quad \text { strongly in } L^{2}\left(\Omega \times Y_{1}\right)^{N}
$$

Moreover, since $e_{i j}\left(\varepsilon \omega^{\alpha} \psi^{\alpha \varepsilon}\right)(x)=\varepsilon \psi_{i}^{\alpha}(x / \varepsilon) e_{i j}\left(\omega^{\alpha}\right)(x)+\omega_{i}^{\alpha}(x) e_{i j}^{y}\left(\psi^{\alpha}\right)(x / \varepsilon)$, for $\alpha \in\{1,2\}$, it is easy to see that

$$
\mathcal{T}_{\alpha}^{\varepsilon}\left(e_{i j}\left(\varepsilon \omega^{\alpha} \psi^{\alpha \varepsilon}\right)\right)=\varepsilon \psi_{i}^{\alpha} \mathcal{T}_{\alpha}^{\varepsilon}\left(e_{i j}\left(\omega^{\alpha}\right)\right)+e_{i j}^{y}\left(\psi^{\alpha}\right) \mathcal{T}_{\alpha}^{\varepsilon}\left(\omega_{i}^{\alpha}\right) \longrightarrow e_{i j}^{y}\left(\Phi^{\alpha}\right)
$$

strongly in $L^{2}\left(\Omega \times Y_{\alpha}\right)$, where $\Phi_{i}^{\alpha}(x, y)=\omega_{i}^{\alpha}(x) \psi_{i}^{\alpha}(y)$ (no summation).

Applying the unfolding operator to $I_{1}^{\varepsilon}$, we obtain that

$$
I_{1}^{\varepsilon}=\int_{\Omega \times Y_{1}} \mathcal{T}_{1}^{\varepsilon}\left(a_{i j k h}^{\varepsilon}\right) \mathcal{T}_{1}^{\varepsilon}\left(e_{k h}\left(u^{1 \varepsilon}\right)\right)\left(\mathcal{T}_{1}^{\varepsilon}\left(e_{i j}(\varphi)\right)+\mathcal{T}_{2}^{\varepsilon}\left(e_{i j}\left(\varepsilon \omega^{1} \psi^{1 \varepsilon}\right)\right)\right) \mathrm{d} x \mathrm{~d} y,
$$

thus, using (5.1) $)_{5}$ and (5.8), we get

$$
I_{1}^{\varepsilon} \longrightarrow \int_{\Omega \times Y_{1}} a_{i j k h}(y)\left(e_{k h}\left(u^{1}\right)+e_{k h}^{y}\left(\hat{u}^{1}\right)\right)\left(e_{i j}(\varphi)+e_{i j}^{y}\left(\Phi^{1}\right)\right) \mathrm{d} x \mathrm{~d} y .
$$


Similarly, by unfolding, we have

$$
I_{2}^{\varepsilon}=\int_{\Omega \times Y_{2}} \mathcal{T}_{2}^{\varepsilon}\left(a_{i j k h}^{\varepsilon}\right) \varepsilon \mathcal{T}_{2}^{\varepsilon}\left(e_{k h}\left(u^{2 \varepsilon}\right)\right) \mathcal{T}_{2}^{\varepsilon}\left(e_{i j}\left(\varepsilon \omega^{2} \psi^{2 \varepsilon}\right)\right) \mathrm{d} x \mathrm{~d} y,
$$

and using $(5.1)_{6}$ and (5.8) yields

$$
I_{2}^{\varepsilon} \longrightarrow \int_{\Omega \times Y_{2}} a_{i j k h}(y) e_{k h}^{y}\left(\hat{u}^{2}\right) e_{i j}^{y}\left(\Phi^{2}\right) \mathrm{d} x \mathrm{~d} y .
$$

Also, from Lemma 9, we get

$$
I_{3}^{\varepsilon}=\int_{\Omega \times \Gamma} h(y)\left(\mathcal{T}_{2}^{\varepsilon}\left(u_{i}^{2 \varepsilon}\right)-\mathcal{T}_{1}^{\varepsilon}\left(u_{i}^{1 \varepsilon}\right)\right)\left(\psi_{i}^{2} \mathcal{T}_{2}^{\varepsilon}\left(\omega_{i}^{2}\right)-\mathcal{T}_{1}^{\varepsilon}\left(\varphi_{i}\right)\right) \mathrm{d} x \mathrm{~d} y
$$

so that from $(5.1)_{2,3}$

$$
I_{3}^{\varepsilon} \longrightarrow \int_{\Omega \times \Gamma} h(y)\left(\hat{u}_{i}^{2}-u_{i}^{1}\right)\left(\psi_{i}^{2} \omega_{i}^{2}-\varphi_{i}\right) \mathrm{d} x \mathrm{~d} y
$$

On the other hand, again Lemma 9 yields

$$
\begin{aligned}
I_{4}^{\varepsilon} & =\varepsilon \int_{\Omega \times \Gamma} h(y)\left(\mathcal{T}_{2}^{\varepsilon}\left(u_{i}^{2 \varepsilon}\right)-\mathcal{T}_{1}^{\varepsilon}\left(u_{i}^{1 \varepsilon}\right)\right) \mathcal{T}_{1}^{\varepsilon}\left(\omega_{i}^{1}\right) \psi_{i}^{1} \mathrm{~d} x \mathrm{~d} y \\
& \leq \varepsilon C \cdot\left\|\mathcal{T}_{2}^{\varepsilon}\left(u_{i}^{2 \varepsilon}\right)-\mathcal{T}_{1}^{\varepsilon}\left(u_{i}^{1 \varepsilon}\right)\right\|_{L^{2}(\Omega \times \Gamma)} \cdot\left\|\mathcal{T}_{1}^{\varepsilon}\left(\omega_{i}^{1}\right) \psi_{i}^{1}\right\|_{L^{2}(\Omega \times \Gamma)},
\end{aligned}
$$

and from $(4.2)_{3}$ we have $I_{4}^{\varepsilon} \leq \varepsilon C$. Consequently,

$$
I_{4}^{\varepsilon} \longrightarrow 0
$$

Finally, we apply the appropriate unfolding operator to $I_{5}^{\varepsilon}, I_{6}^{\varepsilon}, I_{7}^{\varepsilon}$ and for $I_{6}^{\varepsilon}$ we make use of (5.7). Thus,

$$
I_{5}^{\varepsilon} \longrightarrow \int_{\Omega \times Y_{1}} g_{i} \varphi_{i} \mathrm{~d} x \mathrm{~d} y, \quad I_{6}^{\varepsilon} \longrightarrow 0 \quad \text { and } \quad I_{7}^{\varepsilon} \longrightarrow \int_{\Omega \times Y_{2}} g_{i} \Phi_{i}^{2} \mathrm{~d} x \mathrm{~d} y .
$$

The last thing to do in order to obtain the limit problem is to sum up (5.10)-(5.13) and use the density of $\mathcal{D}(\Omega)$ in $H_{0}^{1}(\Omega)$, that of $\mathcal{D}\left(\Omega ; H_{\text {per }}^{1}\left(Y_{1}\right)\right)$ in $L^{2}\left(\Omega ; H_{\text {per }}^{1}\left(Y_{1}\right)\right)$ and the density of $\mathcal{D}\left(\Omega ; H^{1}\left(Y_{2}\right)\right)$ in $L^{2}\left(\Omega ; H^{1}\left(Y_{2}\right)\right)$.

To conclude the proof, observe that endowing the Hilbert space $V$ with the norm

$$
\begin{aligned}
\left\|\left(\varphi, \Phi^{1}, \Phi^{2}\right)\right\|_{V}^{2}= & \left\|\nabla \varphi_{i}\right\|_{L^{2}(\Omega)}^{2}+\left\|\nabla_{y} \Phi_{i}^{1}\right\|_{L^{2}\left(\Omega \times Y_{1}\right)}^{2} \\
& +\left\|\nabla_{y} \Phi_{i}^{2}\right\|_{L^{2}\left(\Omega \times Y_{2}\right)}^{2}+\left\|\Phi_{i}^{2}-\varphi_{i}\right\|_{L^{2}(\Omega \times \Gamma)}^{2},
\end{aligned}
$$

the uniqueness of the solution of problem (5.2) can be proved applying the Lax-Milgram theorem with the norm (5.14) and the bilinear form defined by the left-hand side of (5.2). 
We introduce now, for $l, m=1, \ldots, N$, the unique solutions $w_{1}^{l m} \in \tilde{H}_{\mathrm{per}}^{1}\left(Y_{1}\right)^{N}$ of the problems

$$
\begin{cases}-\frac{\partial}{\partial y_{j}}\left(a_{i j l m}+a_{i j k h} \frac{\partial w_{1 k}^{l m}}{\partial y_{h}}\right)=0 & \text { in } Y_{1}, \\ \left(a_{i j l m}+a_{i j k h} \frac{\partial w_{1 k}^{l m}}{\partial y_{h}}\right) n_{j}=0 & \text { on } \Gamma,\end{cases}
$$

and for $l=1, \ldots, N$, the unique solutions $w_{2}^{l} \in H^{1}\left(Y_{2}\right)^{N}$ of the problems

$$
\left\{\begin{array}{l}
-\frac{\partial}{\partial y_{j}}\left(a_{i j k h} \frac{\partial w_{2 k}^{l}}{\partial y_{h}}\right)=\delta_{i l} \quad \text { in } Y_{2} \\
a_{i j k h} \frac{\partial w_{2 k}^{l}}{\partial y_{h}} n_{j}=h w_{2 i}^{l} \quad \text { on } \Gamma
\end{array}\right.
$$

and finally, we define the homogenized coefficients,

$$
a_{i j l m}^{*}=\int_{Y_{1}}\left(a_{i j l m}+a_{i j k h} \frac{\partial w_{1 k}^{l m}}{\partial y_{h}}\right) \mathrm{d} y .
$$

One can verify that if $\left(u^{1}, \hat{u}^{1}, \hat{u}^{2}\right) \in V$ is the unique solution of (5.2), then

$$
\begin{aligned}
& \hat{u}_{k}^{1}(x, y)=w_{1 k}^{l m}(y) \cdot \frac{\partial u_{l}^{1}}{\partial x_{m}}(x) \quad \text { in } \Omega \times Y_{1}, \\
& \hat{u}_{k}^{2}(x, y)=u_{k}^{1}(x)+g_{l}(x) w_{2 k}^{l}(y) \quad \text { in } \Omega \times Y_{2} .
\end{aligned}
$$

Theorem 12 If $u^{\varepsilon} \in W_{\varepsilon}$ is the solution of (3.10), then

$$
\begin{aligned}
& \tilde{u}^{1 \varepsilon} \rightarrow\left|Y_{1}\right| \cdot u^{1} \quad \text { weakly in } L^{2}(\Omega)^{N}, \\
& \tilde{u}^{2 \varepsilon} \rightarrow\left|Y_{2}\right| \cdot u^{1}+g_{l} \cdot q^{l} \quad \text { weakly in } L^{2}(\Omega)^{N},
\end{aligned}
$$

where $u^{1}$ is the unique solution of the problem

$$
\left\{\begin{array}{l}
-\frac{\partial}{\partial x_{j}}\left(a_{i j k h}^{*} \frac{\partial u_{k}^{1}}{\partial x_{h}}\right)=g_{i} \quad \text { in } \Omega \\
u=0 \text { on } \partial \Omega
\end{array}\right.
$$

and the components of $q^{l}$ are given by $q_{i}^{l}=\int_{Y_{2}} w_{2 i}^{l} \mathrm{~d} y$.

Proof Using suggestive notations, the homogenized problem (5.2) can be written

$$
J_{1}+J_{2}+J_{3}=J_{4}+J_{5}
$$

We will study now each term of (5.23).

From (5.18) we have

$$
\begin{aligned}
J_{1}= & \int_{\Omega} \frac{\partial u_{l}^{1}}{\partial x_{m}} \frac{\partial \varphi_{i}}{\partial x_{j}} \int_{Y_{1}}\left(a_{i j l m}+a_{i j k h} \frac{\partial w_{1 k}^{l m}}{\partial y_{h}}\right) \mathrm{d} x \mathrm{~d} y \\
& +\int_{\Omega} \frac{\partial u_{l}^{1}}{\partial x_{m}} \int_{Y_{1}}\left(a_{i j l m}+a_{i j k h} \frac{\partial w_{1 k}^{l m}}{\partial y_{h}}\right) \frac{\partial \Phi_{i}^{1}}{\partial y_{j}} \mathrm{~d} x \mathrm{~d} y
\end{aligned}
$$


and taking into account (5.17) formula and the variational formulation of (5.15), we get

$$
J_{1}=\int_{\Omega} a_{i j k h}^{*} \frac{\partial u_{k}^{1}}{\partial x_{h}} \frac{\partial \varphi_{i}}{\partial x_{j}} \mathrm{~d} x .
$$

For $J_{2}$ we use (5.19), thus

$$
J_{2}=\int_{\Omega} g_{l} \int_{Y_{2}} a_{i j k h} \frac{\partial w_{2 k}^{l}}{\partial y_{h}} \frac{\partial \Phi_{i}^{2}}{\partial y_{j}} \mathrm{~d} x \mathrm{~d} y
$$

and an integration by parts yields

$$
J_{2}=\int_{\Omega} g_{l} \int_{Y_{2}}-\frac{\partial}{\partial y_{j}}\left(a_{i j k h} \frac{\partial w_{2 k}^{l}}{\partial y_{h}}\right) \Phi_{i}^{2} \mathrm{~d} x \mathrm{~d} y+\int_{\Omega} g_{l} \int_{\Gamma} a_{i j k h} \frac{\partial w_{2 k}^{l}}{\partial y_{h}} \Phi_{i}^{2} n_{2 j} \mathrm{~d} x \mathrm{~d} \sigma_{y},
$$

where $n_{2}$ is the normal on $\Gamma$ exterior to $Y_{2}$. Using now (5.16) and taking into account that $n_{2}=-n, J_{2}$ becomes

$$
J_{2}=\int_{\Omega \times Y_{2}} g_{i} \Phi_{i}^{2} \mathrm{~d} x \mathrm{~d} y-\int_{\Omega \times \Gamma} h g_{l} w_{2 i}^{l} \Phi_{i}^{2} \mathrm{~d} x \mathrm{~d} \sigma_{y} .
$$

Obviously, using (5.19) and the boundary condition from (5.16), we get

$$
J_{3}=\int_{\Omega \times \Gamma} h g_{l} w_{2 i}^{l} \Phi_{i}^{2} \mathrm{~d} x \mathrm{~d} \sigma_{y}-\int_{\Omega} g_{l} \varphi_{i} \int_{\Gamma} a_{i j k h} \frac{\partial w_{2 k}^{l}}{\partial y_{h}} n_{j} \mathrm{~d} x \mathrm{~d} \sigma_{y},
$$

and, due to Gauss-Ostrogradsky formula and the fact that $n_{2}=-n$,

$$
\begin{aligned}
J_{3} & =\int_{\Omega \times \Gamma} h g_{l} w_{2 i}^{l} \Phi_{i}^{2} \mathrm{~d} x \mathrm{~d} \sigma_{y}+\int_{\Omega} g_{l} \varphi_{i} \int_{Y_{2}} \frac{\partial}{\partial y_{j}}\left(a_{i j k h} \frac{\partial w_{2 k}^{l}}{\partial y_{h}}\right) \mathrm{d} x \mathrm{~d} y \\
& =\int_{\Omega \times \Gamma} h g_{l} w_{2 i}^{l} \Phi_{i}^{2} \mathrm{~d} x \mathrm{~d} \sigma_{y}-\left|Y_{2}\right| \int_{\Omega} g_{i} \varphi_{i} \mathrm{~d} x .
\end{aligned}
$$

Hence (5.23) becomes

$$
\int_{\Omega} a_{i j k h}^{*} \frac{\partial u_{k}^{1}}{\partial x_{h}} \frac{\partial \varphi_{i}}{\partial x_{j}} \mathrm{~d} x-\left|Y_{2}\right| \int_{\Omega} g_{i} \varphi_{i} \mathrm{~d} x=\left|Y_{1}\right| \int_{\Omega} g_{i} \varphi_{i} \mathrm{~d} x
$$

which means that

$$
\int_{\Omega} a_{i j k h}^{*} \frac{\partial u_{k}^{1}}{\partial x_{h}} \frac{\partial \varphi_{i}}{\partial x_{j}} \mathrm{~d} x=\int_{\Omega} g_{i} \varphi_{i} \mathrm{~d} x
$$

and finally (5.22) is proved. The convergences (5.20) and (5.21) are given directly by $(5.1)_{1,2}$. 


\section{Author details}

'Normandie Université, Laboratoire de Mathématiques Raphaël Salem, Université de Rouen, CNRS, UMR 6085, Avenue de I'Université, BP 12, Saint-Étienne du Rouvray cedex, 76801, France. ${ }^{2}$ Simion Stoilow Institute of Mathematics of the Romanian Academy, 21 Calea Grivitei Street, Bucharest, 010702, Romania.

\section{Acknowledgements}

The second author's work was accomplished during his visit to the Raphaël Salem Laboratory of Mathematics of the University of Rouen and it was supported by the Sectorial Operational Programme Human Resources Development (SOP $\mathrm{HRD}$ ), financed from the European Social Fund and by the Romanian Government under the contract number SOP $\mathrm{HRD} / 107 / 1.5 / \mathrm{S} / 82514$. He would like to express his special thanks for the invitation to the laboratory and he is also very grateful to Prof. Horia I Ene for useful discussions regarding the mechanical model studied in the present paper.

\section{Received: 28 August 2013 Accepted: 7 November 2013 Published: 03 Dec 2013}

\section{References}

1. Cioranescu, D, Damlamian, A, Griso, G: Periodic unfolding and homogenization. C. R. Acad. Sci. Paris, Sér. I Math. 335 , 99-104 (2002)

2. Cioranescu, D, Damlamian, A, Griso, G: The periodic unfolding method in homogenization. SIAM J. Math. Anal. 40(4), 1585-1620 (2008)

3. Cioranescu, D, Donato, P, Zaki, R: The periodic unfolding method in perforated domains. Port. Math. 63(4), 467-496 (2006)

4. Cioranescu, D, Damlamian, A, Donato, P, Griso, G, Zaki, R: The periodic unfolding method in domains with holes. SIAM J. Math. Anal. 44(2), 718-760 (2012)

5. Donato, $\mathrm{P}$, Le Nguyen, $\mathrm{KH}$, Tardieu, R: The periodic unfolding method for a class of imperfect transmission problems. J. Math. Sci. (N.Y.) 176(6), 891-927 (2011)

6. Auriault, JL, Ene, HI: Macroscopic modelling of heat transfer in composites with interfacial thermal barrier. Int. J. Heat Mass Transf. 37(18), 2885-2892 (1994)

7. Ene, HI: On the microstructure models of porous media Rev. Roum. Math. Pures Appl. 46(2-3), 289-295 (2001)

8. Ene, HI, Poliševski, D: Model of diffusion in partially fissured media. Z. Angew. Math. Phys. 53(6), 1052-1059 (2002)

9. Nguetseng, G: A general convergence result for a functional related to the theory of homogenization. SIAM J. Math. Anal. 20, 608-629 (1989)

10. Allaire, G: Homogenization and two-scale convergence. SIAM J. Math. Anal. 23(6), 1482-1518 (1992)

11. Monsurrò, S: Homogenization of a two-component composite with interfacial thermal barrier. Adv. Math. Sci. Appl. 13(1), 43-63 (2003)

12. Donato, $\mathrm{P}$, Monsurrò, S: Homogenization of two heat conductors with an interfacial contact resistance. Anal. Appl. 2(3), 247-273 (2004)

13. Poliševski, D: The regularized diffusion in partially fractured media. In: Current Topics in Continuum Mechanics, pp. 106-116. Ed. Academiei, Bucharest (2003)

14. Smyshlyaev, VP: Propagation and localization of elastic waves in highly anisotropic periodic composites via two-scale homogenization. Mech. Mater. 41, 434-447 (2009)

15. Cioranescu, D, Damlamian, A, Orlik, J: Homogenization via unfolding in periodic elasticity with contact on closed and open cracks. Asymptot. Anal. 82(3-4), 201-232 (2013)

16. Orlik, J: Two-scale homogenization in transmission problems of elasticity with interface jumps. Appl. Anal. 91(7), 1299-1319 (2012)

17. Duvaut, G: Analyse fonctionnelle et mécanique des milieux continus. Applications à l'étude des matériaux composites élastiques à structure périodique, homogénéisation. In: Koiter, WT (ed.) Theoretical and Applied Mechanics. North-Holland, Amsterdam (1978)

18. Sanchez-Palencia, E: Non-Homogeneous Media and Vibration Theory. Lecture Notes in Physics, vol. 127. Springer, Berlin (1980)

19. Cioranescu, D, Donato, P: An Introduction to Homogenization. Oxford Lecture Series in Mathematics and Its Applications, vol. 17 (1999)

20. Oleinik, OA, Shamaev, AS, Yosifian, GA: Mathematical Problems in Elasticity and Homogenization. North-Holland, Amsterdam (1992)

21. Léné, F: Comportement macroscopique de matériaux élastiques comportant des inclusions rigides ou des trous répartis périodiquement. C. R. Acad. Sci. Paris, Sér. I Math. 292, 75-78 (1981)

22. Cioranescu, D, Saint Jean-Paulin, J: Homogenization in open sets with holes. J. Math. Anal. Appl. 71, 590-607 (1979)

23. Ciarlet, PG: An Introduction to Differential Geometry with Applications to Elasticity. Reprinted from J. Elasticity 78/79 (2005), no. 1-3 [MR2196098]. Springer, Dordrecht (2005)

24. Duvaut, G, Lions, J-L: Les inéquations en mécanique et en physique. Dunod, Paris (1972)

25. Kristaly, A, Radulescu, V, Varga, C: Variational Principles in Mathematical Physics, Geometry, and Economics. Qualitative Analysis of Nonlinear Equations and Unilateral Problems. Encyclopedia of Mathematics and Its Applications, vol. 136. Cambridge University Press, Cambridge (2010)

10.1186/1687-2770-2013-265

Cite this article as: Donato and Tenţea: Homogenization of an elastic double-porosity medium with imperfect interface via the periodic unfolding method. Boundary Value Problems 2013, 2013:265 